\title{
Metabolomic Foot Printing and Microbial Pathogens
}

\section{Schlegel Vl*}

${ }^{1}$ Department of Food Science and Technology, University of Nebraska-Lincoln, Lincoln, NE, USA

\section{Introduction}

Metabolomic foot printing has evolved in its own right as a powerful approach to understand the effects of environment/genetic signals on biological systems [1,2]. Unlike classical metabolomics (or) metabolomics fingerprinting), where the internal metabolome of cell or tissue is defined, the goal of metabolomics foot printing is to characterize the low molecular weight metabolites excreted/secreted by and/or consumed by the system [3]. The complement of external metabolites identified by foot printing methods is thus referred to as the metabolomic footprint, exometabolome or secretome, albeit the latter also includes the proteome [4]. Compared to classical metabolomics, advantages to metabolomics foot printing is the elimination of fast metabolism quenching steps and time consuming extraction procedures, while applying similar identification/quantitation techniques used for classical metabolomics, such as LC-MS, GC-MS, NMR, and vibrational spectroscopies and data mining analyses.

Microbial pathogens are responsible for such infections that include sepsis, cholera, tuberculosis, which can also lead to cancers other diseases. As all biological systems (cells, tissues, plants, etc.) secrete and consume metabolites in response to their environment, their metabolomic footprint provides additional insight into a system's internal metabolic activity. Application of metabolomics foot printing to microbial pathogens is especially important considering that their virulence is predicated on cues from the environment. For example, pathogens rely on signaling components to sense their environment and thereby respond accordingly to available nutrients and other microbial populations. Metabolomic foot printing can thus be used to monitor these signaling molecules as a means to prevent the virulence switch in otherwise benign organisms. Alternatively a nontargeted approach may be used correlate the potential of virulence, such as inoculation, growth, and/or colonization, and prevent such infections before they occur. But, and most importantly, combining the metabolomics footprints with its fingerprint counterpart can elucidate the mechanisms that drive the pathogenic response.

More specifically, Carneiro et al. [5], evaluated the metabolomic footprints of Helicobacter pylori, and revealed that six key amino acids were needed for its growth. Additionally, Hoerr et al. [6] combined the results of Pseudomonas aeruginosa footprints obtained from culture experiments with a mouse study to determine that the levels of a variety of amino acids were increased, while metabolites from anaerobic and aerobic pathways were not detected. The authors concluded from these results could be used to directly identify the infecting organism for diagnostic purposes. In our own studies, significant differences resulted in the footprints of Candida albicans as it transitioned from its non-virulent yeast to its virulent hyphae phenotype, as determined by multivariate analyses of FT-IR spectra (unpublished results). Interestingly, the footprints showed evidence of the virulent phenotype prior to a change in its morphology. We are now using this information as a means to develop effective natural anti-fungal agents for preventing the pathogenic switch without threatening C. albicans existence. Lastly, Liebeke et al. [7] examined both the footprints and fingerprints of Staphylococcus aureus under starvation and excess glucose conditions. By coupling these techniques, the researchers showed that glycolysis was extremely active when exposed to excess carbon growth with multiple glycolytic intermediates overflowing into the media. However, during starvation conditions, glycolysis was suppressed and the overflow glycolytic intermediates were then consumed. The reverse occurred for the citric acid cycle, indicating an anabolic pathway. Such studies can thus be used to determine treatments that account for the differences in pathogens' metabolism when exposed to their ever changing environments. In summary, these limited studies demonstrate the scope of metabolomic fingerprinting for understanding the pathogenic response, which strengthens our ability to develop resistant antimicrobial compounds.

\section{References}

1. Suhre K, Meisinger C, Döring A, Altmaier E, Belcredi P, et al. (2010) Metabolic Footprint of Diabetes: A Multiplatform Metabolomics Study in an Epidemiological Setting. PLoS ONE 5: e13953.

2. Kell DB, Brown M, Davey HM, Dunn WB, Spasic I (2005) Metabolic footprinting and systems biology: the medium is the message. Nat Rev Microbiol 3: 557 565

3. Mapelli V, Olsson L, Nielsen J (2008) Metabolic footprinting in microbiology: methods and applications in functional genomics and biotechnology. Trends Biotechnol 26: 490-497.

4. Greenbaum D, Luscombe NM, Jansen R, Qian J, Gerstein M (2001) Interrelating different types of genomic data from proteome to secretome: oming in on function. Genome Res 11: 1463-1468.

5. Carneiro S, Correia Daniela M, Ferreira EC, Rocha I (2012) Footprinting microbial metabolites in nature and medicine. SYSMED 2012 - International Conference on Systems Medicine. Dublin, Irlande, pp. 63-65.

6. Hoerr V, Zbytnuik L, Leger C, Tam PP, Kubes P, et al. (2012) Gram-negative and gram positive bacterial infections give rise to a different metabolic response in a mouse model. J Proteome Res 11: 3231-3245

7. Liebeke M, Dörries K, Zühlke D, Bernhardt J, Fuchs S, et al. (2011) A metabolomics and proteomics study of the Staphlococcus aureus to glucose starvation. Mol Biosyst 7: 1241-1253.

*Corresponding author: Schlegel V, Department of Food Science and Technology, University of Nebraska-Lincoln, Lincoln, NE, USA, Tel: 402-416-0294; Fax: 402- 4721693; E-mail: vschlegel3@unl.edu

Received: February 01, 2015; Accepted: February 01, 2015; Published: February 04, 2015

Citation: Schlegel VI (2015) Metabolomic Foot printing and Microbial Pathogens. Metabolomics 5: e132. doi:10.4172/2153-0769.1000e132

Copyright: (c) 2015 Schlegel VI. This is an open-access article distributed unde the terms of the Creative Commons Attribution License, which permits unrestricted use, distribution, and reproduction in any medium, provided the original author and source are credited. 